\title{
A NOTE ON VON NEUMANN RHO-INVARIANT OF SURFACE BUNDLES OVER THE CIRCLE
}

\author{
TAKAYUKI MORIFUJI
}

(Received March 8, 2004, revised August 6, 2004)

\begin{abstract}
In this short note, we give a formula for the von Neumann rho-invariant of surface bundles over the circle $S^{1}$. As a corollary, we describe a relation among the von Neumann rho-invariant, the first Morita-Mumford class and the Rochlin invariant in a framework of the bounded cohomology.
\end{abstract}

1. Introduction. Let $M$ be an oriented closed Riemannian 3-manifold. Then we can define the $\eta$-invariant $\eta(M)$ of the signature operator. If we are given a surjective homomorphism from $\pi_{1} M$ to a discrete group $\Gamma$, we have a $\Gamma$-covering $\bar{M} \rightarrow M$ and we can lift the metric and the signature operator to $\bar{M}$. In this situation, the von Neumann $\eta$-invariant $\eta^{(2)}(\bar{M})$ is defined for $\bar{M}$. Cheeger and Gromov showed in [3] that the difference $\eta^{(2)}-\eta$ is independent of a Riemannian metric. This topological invariant $\eta^{(2)}-\eta$ is called the von Neumann rho-invariant and is denoted by $\rho^{(2)}(\bar{M})$.

Recently, an approximation theorem of the $\eta$-invariants is shown by Vaillant [11] and Lück-Schick [7]. To be more precise, for a sequence of normal subgroups $\Gamma \triangleright \Gamma_{1} \triangleright \Gamma_{2} \triangleright \cdots$ such that $\left[\Gamma: \Gamma_{k}\right]<\infty$ and $\bigcap_{k} \Gamma_{k}=\{e\}$, and $\Gamma / \Gamma_{k}$-coverings $M^{k}=\bar{M} / \Gamma_{k} \rightarrow M$, it holds that

$$
\eta^{(2)}(\bar{M})=\lim _{k \rightarrow \infty} \frac{\eta\left(M^{k}\right)}{\left[\Gamma: \Gamma_{k}\right]} .
$$

Applying this formula to surface bundles over the circle $S^{1}$, we can describe the von Neumann rho-invariant by virtue of Meyer's signature cocycle [8] (see Proposition 2.1).

Let $\Sigma_{g}$ be an oriented closed surface of genus $g(g \geq 2)$ and $\mathcal{M}_{g}$ its mapping class group. Namely, $\mathcal{M}_{g}$ is the group of all isotopy classes of orientation preserving diffeomorphisms of $\Sigma_{g}$. Then the first Morita-Mumford class $e_{1} \in H^{2}\left(\mathcal{M}_{g}, \boldsymbol{Z}\right)$ (see [10]) is defined to be the Gysin image (integration along the fiber) of the square of the Euler class of the central extension

$$
0 \rightarrow Z \rightarrow \mathcal{M}_{g, 1} \rightarrow \mathcal{M}_{g, *} \rightarrow 1
$$

Here $\mathcal{M}_{g, 1}$ is the mapping class group of $\Sigma_{g}$ relative to an embedded disc $D \subset \Sigma_{g}$ and $\mathcal{M}_{g, *}$ denotes the one relative to a base point $* \in D$. It is also known that $e_{1}$ is a bounded

2000 Mathematics Subject Classification. Primary 57M27; Secondary 57R20.

Key words and phrases. von Neumann rho-invariant, Morita-Mumford class, Rochlin invariant, bounded cohomology.

Supported in part by the Grand-in-Aid for Scientific Research (No. 14740036), The Ministry of Education, Culture, Sports, Science and Technology, Japan. 
cohomology class. For a technical reason (see Section 3), we consider $e_{1}$ on $\mathcal{M}_{g, *}$ rather than on $\mathcal{M}_{g}$.

In general, the pull back of $e_{1}$ via a holonomy homomorphism $f: \pi_{1} S^{1} \rightarrow \mathcal{M}_{g, *}$ of a $\Sigma_{g}$-bundle over $S^{1}$ is automatically vanishing, because $H^{2}\left(S^{1}, \boldsymbol{Z}\right)$ is trivial. However, Kitano showed in [6] that $e_{1} / 48$ makes sense as a bounded cohomology class in $H_{b}^{2}\left(S^{1}, \boldsymbol{Z}\right)$ and furthermore it is essentially given by the Rochlin invariant $\mu$, if the image of $f$ is contained in the Torelli subgroup $\mathcal{I}_{g, *}=\operatorname{Ker}\left\{\mathcal{M}_{g, *} \rightarrow \operatorname{Sp}(2 g, \boldsymbol{Z})\right\}$. Combining our formula for $\rho^{(2)}$ with a result of Kitano, we have the following result on the level 2 subgroup $\mathcal{M}_{g, *}(2)=$ $\operatorname{Ker}\left\{\mathcal{M}_{g, *} \rightarrow \operatorname{Sp}(2 g, \boldsymbol{Z} / 2)\right\} \supset \mathcal{I}_{g, *}$

THEOREM 1.1. Let $f: \boldsymbol{Z} \rightarrow \mathcal{M}_{g, *}(2)$ be a holonomy homomorphism. Then the first Morita-Mumford class $f^{*} e_{1} / 48 \in H_{b}^{2}(\mathbf{Z}, \mathbf{Z})$ is represented by $\mu f-\rho^{(2)} f / 16 \in H^{1}(\boldsymbol{Z}, \boldsymbol{R} / \mathbf{Z})$.

REMARK 1.2. It is known that $H_{b}^{2}(\boldsymbol{Z}, \boldsymbol{Z}) \cong H_{b}^{1}(\boldsymbol{Z}, \boldsymbol{R} / \boldsymbol{Z}) \cong H^{1}(\boldsymbol{Z}, \boldsymbol{R} / \boldsymbol{Z}) \cong \boldsymbol{R} / \boldsymbol{Z}$. See [4], [6] for the proof.

As was mentioned in [6], $e_{1} / 48$ and $\mu$ depend on a fixed spin structure on the fiber $\Sigma_{g}$. In principle, our theorem implies that the difference between the first Morita-Mumford class and the Rochlin invariant does not depend on a spin structure, and it is given by the von Neumann rho-invariant. In particular, we see from Corollary 2.4 that vanishing of the von Neumann rho-invariant gives a description of the first Morita-Mumford class via the Rochlin invariant on the Torelli group $\mathcal{I}_{g, *}$.

The final version of this note was written while the author was visiting the LudwigMaximilians-Universität in München. He would like to express his sincere thanks for their hospitality.

2. A formula of $\rho^{(2)}$. In this section, we give a formula for the von Neumann rhoinvariant $\rho^{(2)}$ of surface bundles over $S^{1}$. As for the precise definition, see Cheeger-Gromov [3]. We remark that it does not depend on a Riemannian metric on the manifold.

Let $M_{\varphi}$ be the mapping torus $\Sigma_{g} \times \boldsymbol{R} /(x, t) \sim(\varphi(x), t+1)$ corresponding to a holonomy $\varphi \in \mathcal{M}_{g, *}$. Let $\boldsymbol{Z} \rightarrow \bar{M}_{\varphi} \rightarrow M_{\varphi}$ be the $\boldsymbol{Z}$-covering associated to the surjective homomorphism $p: \pi_{1} M_{\varphi} \rightarrow \pi_{1} S^{1} \cong \boldsymbol{Z}$.

PROPOSITION 2.1. The von Neumann rho-invariant of $\bar{M}_{\varphi}$ is given by

$$
\rho^{(2)}\left(\bar{M}_{\varphi}\right)=-\lim _{k \rightarrow \infty} \frac{1}{k} \sum_{i=1}^{k-1} \operatorname{sign}\left(\varphi, \varphi^{i}\right),
$$

where sign is Meyer's signature 2-cocycle [8] of the mapping class group $\mathcal{M}_{g, *}$.

Proof. Set $M_{\varphi}^{k}=\bar{M}_{\varphi} / k ! \boldsymbol{Z}$. It is easy to see that $\boldsymbol{Z} / k ! \rightarrow M_{\varphi}^{k} \rightarrow M_{\varphi}$ is the $\boldsymbol{Z} / k !-$ covering associated to a homomorphism $p_{k}: \pi_{1} M_{\varphi} \rightarrow \boldsymbol{Z} / k$ !. We then apply an approximation theorem of the $\eta$-invariants, due to Vaillant [11] and Lück-Schick [7], to the sequence $Z_{\triangleright} 2 ! Z_{\triangleright} 3 ! Z_{\triangleright} \cdots$. It follows that 


$$
\begin{aligned}
\rho^{(2)}\left(\bar{M}_{\varphi}\right) & =\eta^{(2)}\left(\bar{M}_{\varphi}\right)-\eta\left(M_{\varphi}\right)=\lim _{k \rightarrow \infty} \frac{\eta\left(M_{\varphi}^{k}\right)}{k !}-\eta\left(M_{\varphi}\right) \\
& =\lim _{k \rightarrow \infty}\left\{\frac{1}{k !} \eta\left(M_{\varphi^{k !}}\right)-\eta\left(M_{\varphi}\right)\right\} .
\end{aligned}
$$

Since $M_{\varphi^{k}} \rightarrow M_{\varphi}$ is a $k$ !-fold cyclic covering, we can directly apply our previous results to this covering (see [9] Propositions 2.2 and 3.1). Hence we obtain

$$
\rho^{(2)}\left(\bar{M}_{\varphi}\right)=\lim _{k \rightarrow \infty} \frac{1}{3 k !} \sum_{i=1}^{k !-1} c\left(\varphi, \varphi^{i}\right),
$$

where $c$ denotes Atiyah's 2-cocycle [1] of the mapping class group defined by the canonical 2 -framing of 3-manifolds. Furthermore it is known that the cocycle $c$ coincides with $-3 \times$ Meyer's signature cocycle sign (see [10] for instance). Therefore we have

$$
\rho^{(2)}\left(\bar{M}_{\varphi}\right)=-\lim _{k \rightarrow \infty} \frac{1}{k !} \sum_{i=1}^{k !-1} \operatorname{sign}\left(\varphi, \varphi^{i}\right)=-\lim _{k \rightarrow \infty} \frac{1}{k} \sum_{i=1}^{k-1} \operatorname{sign}\left(\varphi, \varphi^{i}\right) .
$$

Remark 2.2. By definition, Meyer's cocycle is a bounded 2-cocycle, so that the above limit exists. Moreover this defines a class function on $\mathcal{M}_{g, *}$. We also remark that the above formula holds for torus bundles (that is, $g=1$ ).

EXAMPLE 2.3. Let us consider the genus one case. As is well-known, in this case $\mathcal{M}_{1, *} \cong S L(2, \boldsymbol{Z})$ holds. An element $A \in S L(2, \boldsymbol{Z})$ is classified by its trace into the following three cases:

(i) Elliptic case (namely, $|\operatorname{tr} A|<2)$. Let $A_{n} \in S L(2, \boldsymbol{Z})$ have the order $n$ ( $n=$ $3,4,6)$. We can take

$$
A_{3}=\left(\begin{array}{cc}
-1 & -1 \\
1 & 0
\end{array}\right), \quad A_{4}=\left(\begin{array}{cc}
0 & -1 \\
1 & 0
\end{array}\right) \quad \text { and } \quad A_{6}=\left(\begin{array}{cc}
0 & -1 \\
1 & 1
\end{array}\right) .
$$

An easy calculation shows that $\operatorname{sign}\left(A_{n}, A_{n}\right)=\cdots=\operatorname{sign}\left(A_{n}, A_{n}^{n-2}\right)=-2$ and $\operatorname{sign}\left(A_{n}, A_{n}^{n-1}\right)=\operatorname{sign}\left(A_{n}, A_{n}^{n}\right)=0$ (see [8]). Hence we have

$$
\rho^{(2)}\left(\bar{M}_{A_{n}}\right)= \begin{cases}2 / 3, & n=3 \\ 1, & n=4, \\ 4 / 3, & n=6 .\end{cases}
$$

It should be noted that $\rho^{(2)}\left(\bar{M}_{\varphi}\right)=0$ for any involution $\varphi \in \mathcal{M}_{g, *}$ (see [9]).

(ii) Parabolic case (namely, $|\operatorname{tr} A|=2$ ). We can take

$$
A_{b}=\left(\begin{array}{ll}
1 & b \\
0 & 1
\end{array}\right) \quad(b \in \boldsymbol{Z}) .
$$

Then we obtain $\rho^{(2)}\left(\bar{M}_{A_{b}}\right)=-\operatorname{sgn}(b)$, where $\operatorname{sgn}(b)=b /|b|$ if $b \neq 0$ and 0 if $b=0$.

(iii) Hyperbolic case (namely, $|\operatorname{tr} A|>2$ ). Since Meyer's function of genus one, that is a class function $\phi: S L(2, \boldsymbol{Z}) \rightarrow(1 / 3) \boldsymbol{Z}$ such that $\delta \phi=$ sign, satisfies $\phi\left(A^{k}\right)=k \phi(A)$ for 
a hyperbolic element $A$ (see [8]), we have

$$
\rho^{(2)}\left(\bar{M}_{A}\right)=-\lim _{k \rightarrow \infty} \frac{1}{k} \sum_{i=1}^{k-1} \operatorname{sign}\left(A, A^{i}\right)=-\lim _{k \rightarrow \infty}\left\{\phi(A)-\frac{1}{k} \phi\left(A^{k}\right)\right\}=0 .
$$

COROLLARY 2.4. If $\varphi$ is an element of the Torelli group $\mathcal{I}_{g, *}$, then $\rho^{(2)}\left(\bar{M}_{\varphi}\right)=0$.

Proof. Meyer's 2-cocycle sign is originally defined on the Siegel modular group $\operatorname{Sp}(2 g, \boldsymbol{Z})$, and $\mathcal{I}_{g, *}$ is the kernel of the homomorphism $\mathcal{M}_{g, *} \rightarrow \operatorname{Sp}(2 g, \boldsymbol{Z})$, so that $\operatorname{sign}\left(\varphi, \varphi^{i}\right)$ vanishes for any $i$.

3. Morita-Mumford class and Rochlin invariant. In this section, we summarize a work of Kitano [6], which gives a description of the Rochlin invariant as a secondary characteristic class within a framework of the bounded cohomology $H_{b}^{*}$. As for the definition of $H_{b}^{*}$, see Gromov [5].

Let $(M, \alpha)$ be an oriented spin 3-manifold with a spin structure $\alpha$. It is a classical result that there exists a compact oriented spin 4-manifold $(W, \beta)$ such that $\partial W=M$ and $\left.\beta\right|_{M}=\alpha$. The Rochlin invariant $\mu(M, \alpha) \in \boldsymbol{Q} / \boldsymbol{Z}$ is defined by

$$
\mu(M, \alpha)=\frac{\operatorname{Sign} W}{16} \bmod \boldsymbol{Z},
$$

where Sign $W$ denotes the signature of a 4-manifold $W$. By Rochlin's theorem, $\mu(M, \alpha)$ does not depend on the choice of $W$.

Let us fix a spin structure $\alpha$ of $\Sigma_{g}(g \geq 2)$. For each $\varphi \in \mathcal{M}_{g, *}(2)$, there exists a spin structure $\tilde{\alpha}$ on $M_{\varphi}$ such that the restriction on each fiber is $\alpha$. If we require that the restriction of $\tilde{\alpha}$ to the $S^{1}$-orbit of $* \in \Sigma_{g}$ is the bounding spin structure (namely, not the Lie group spin structure), then $\tilde{\alpha}$ is uniquely determined. This is the reason why we consider $\mathcal{M}_{g, *}(2)$ rather than $\mathcal{M}_{g}$.

Now consider the set of pairs $\{(\varphi, W)\}$, where $\varphi \in \mathcal{M}_{g, *}(2)$ and $W$ is an oriented spin 4-manifold. Of course, $\partial W=M_{\varphi}$ and the induced spin structure on $M_{\varphi}$ is $\tilde{\alpha}$. Two pairs $(\varphi, W)$ and $\left(\varphi, W^{\prime}\right)$ are said to be equivalent if Sign $W=\operatorname{Sign} W^{\prime}$. The set of equivalence classes, which we denote by $\mathcal{M}_{g, *}^{\alpha}(2)$, has a group structure defined by the fiber connected sum (see [6] for details), and there is a natural surjective homomorphism

$$
\mathcal{M}_{g, *}^{\alpha}(2) \rightarrow \mathcal{M}_{g, *}(2)
$$

given by $(\varphi, W) \mapsto \varphi$. Moreover we introduce a map $\tau: \mathcal{M}_{g, *}^{\alpha}(2) \rightarrow \boldsymbol{Q}$ by

$$
\tau(\varphi, W)=\frac{1}{16} \text { Sign } W \in \frac{1}{16} \boldsymbol{Z} \subset \boldsymbol{Q} .
$$

PROPOSITION 3.1 (Kitano [6]). Under the setting above, the following hold.

(i) The Euler class $e_{\alpha}$ of the extension $\mathcal{M}_{g, *}^{\alpha}(2) \rightarrow \mathcal{M}_{g, *}(2)$ is a bounded cohomology class and is given by $e_{1} / 48$ on the level 2 subgroup $\mathcal{M}_{g, *}(2)$.

(ii) Let $f: \boldsymbol{Z} \rightarrow \mathcal{M}_{g, *}(2)$ be a homomorphism. The pull back $f^{*} e_{\alpha} \in H_{b}^{2}(\boldsymbol{Z}, \boldsymbol{Z})$ is described by $\bar{\tau}_{\infty} f \in H^{1}(\boldsymbol{Z}, \boldsymbol{R} / \mathbf{Z})$, where $\bar{\tau}_{\infty}: \mathcal{M}_{g, *}(2) \rightarrow \boldsymbol{R} / \mathbf{Z}$ is the reduction mod $\mathbf{Z}$ of 
a map

$$
\tau_{\infty}(\varphi, W)=\lim _{k \rightarrow \infty} \frac{\tau\left((\varphi, W)^{k}\right)}{k} \in \boldsymbol{R}
$$

From the additivity of the signature, we obtain

$$
\tau_{\infty}(\varphi, W)=\tau(\varphi, W)+\frac{1}{16} \lim _{k \rightarrow \infty} \frac{1}{k} \sum_{i=1}^{k-1} \operatorname{sign}\left(\varphi, \varphi^{i}\right) .
$$

Taking the reduction $\bmod \boldsymbol{Z}$ and using Proposition 2.1, we have

$$
\bar{\tau}_{\infty}(\varphi)=\mu\left(M_{\varphi}, \tilde{\alpha}\right)-\frac{1}{16} \rho^{(2)}\left(\bar{M}_{\varphi}\right) \in \boldsymbol{R} / \boldsymbol{Z}
$$

for $\varphi \in \mathcal{M}_{g, *}(2)$. Therefore Theorem 1.1 follows from Proposition 3.1 and the proof is completed.

\section{REFERENCES}

[ 1 ] M. ATIYAH, On framings of 3-manifolds, Topology 29 (1990), 1-7.

[2] J. Barge And E. Ghys, Cocycles d'Euler et de Maslov, Math. Ann. 294 (1992), 235-265.

[ 3 ] J. CheEger AND M. Gromov, Bounds on the von Neumann dimension of $L^{2}$-cohomology and the GaussBonnet theorem for open manifolds, J. Differential Geom. 21 (1985), 1-34.

[ 4 ] E. GHYs, Groupes d'homéomorphismes du cercle et cohomologie bornée, The Lefschetz centennial conference, III (Mexico City, 1984), 81-106, Contemp. Math. 58, Amer. Math. Soc., Providence, R.I., 1987.

[ 5 ] M. Gromov, Volume and bounded cohomology, Inst. Hautes Études Sci. Publ. Math. 56 (1982), 5-100.

[ 6 ] T. Kitano, On the first Morita-Mumford class of surface bundles over $S^{1}$ and the Rochlin invariant, J. Knot Theory Ramifications 9 (2000), 179-186.

[ 7 ] W. LÜCK AND T. SCHICK, Approximating $L^{2}$-signatures by their finite-dimensional analogues, Forum Math. 17 (2005), 31-65.

[ 8 ] W. MeYer, Die Signatur von Flächenbündeln, Math. Ann. 201 (1973), 239-264.

[ 9 ] T. MoRifujI, The $\eta$-invariant of mapping tori with finite monodromies, Topology Appl. 75 (1997), 41-49.

[10] S. MoRita, Structure of the mapping class groups of surfaces: a survey and a prospect, Proceedings of the Kirbyfest (Berkeley, CA, 1998), 349-406 (electronic), Geom. Topol. Monogr. 2, Geom. Topol. Publ., Coventry, 1999.

[11] B. VAILlant, Indextheorie für Überlagerungen, Diplomarbeit, Universität Bonn, 1997.

DEPARTMENT OF MATHEMATICS

TOKyo University of Agriculture AND TECHNOLOGY

2-24-16 NAKA-CHO

KOGANEI TOKYO 184-8588

JAPAN

E-mail address: morifuji@cc.tuat.ac.jp 